\title{
Preface
}

\section{Why RRS? Where RRS?}

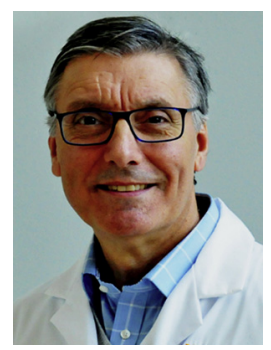

Michael A. DeVita, MD, FCCM, FRCP, FACP

Editor

The concept of rapid response systems (RRSs) was first imagined after a 19-year-old woman bled to death on a general ward of a major teaching hospital. There had been a slow but obvious increase in respiratory and pulse rate, and a decrease in blood pressure. The nurses informed the intern, who informed the resident, who informed the surgeon, who was operating in the operating room. By the time the surgeon saw her, she had arrested. She had slowly exsanguinated. This event resulted in no changes in the process of care. Years later, in Australia, Dr Hillman developed the first RRS, the medical emergency team (MET). It was designed to recognize and respond to deteriorating patients before they suffered serious adverse events. It was a "preemptive strike," in essence sending the "code team" before the code.

The RRS is a process that uses standard objective criteria to define the at-risk patient. It cuts across the usual hospital silos and hierarchies and addresses the needs of the patient with a specialized team. The RRS moves the skills and equipment out of the ICU to create an ICU-like environment anywhere. While most ward clinicians are skillful, they are not there all the time, and they may not be trained in critical illness. The earliest RRS implementers had an uphill battle: they showed that the MET was needed, but in doing so implied that the usual system was a problem. There was significant pushback.

Now rapid response teams are commonplace and often required by governmental or regulatory bodies. How to organize the team is well reported, and there are a number of textbooks available. The RRS has been shown to have value for the treatment of many critical situations, and some hospitals now have a number of different RRTs for a variety of time-critical situations, including "stroke teams," "coronary syndrome teams," and "sepsis teams." They are effective and have saved innumerable lives worldwide.

RRSs share four cornerstones: an ability to detect critical abnormality and trigger a response, a response of equipment and personnel, an analysis of events to help 
prevent future events through usual quality improvement mechanisms, and administrative leadership.

In this Critical Care Clinics, the articles explore ways that the concept of RRSs has now expanded to operate in different settings. The articles have two foci. The first highlights the state-of-the-art in monitoring and triggering. It is increasingly recognized that without a reliable and sensitive detection system, no RRS can possibly exist, let alone succeed. Dr Wong Lama and colleagues describe efforts to organize the gigantic amount of data that exists in the electronic health record to enable analysis and prediction. The work on "big data" is promising. It is hoped it will be able to diagnose syndromes that might be missed or recognized late, including recognizing very early deterioration to prevent the need to respond emergently. Another approach to early detection, reported herein by Dr Taenzer and colleagues, emphasizes the use of continuous physiologic monitoring to "fill in the gaps" that occur when vital signs are obtained intermittently. The gaps can miss deterioration of some patients, leading to disastrous results. We expect that continuous monitoring will someday be a standard of care for all hospitalized patients.

The second cluster of articles relates to some critical, focused applications of the RRS. The situations share a need for speed, organization, and accuracy of care. Dr Sarani describes sepsis teams; Dr Mark explores the difficult airway response team, and Dr Dalby describes the experience with an obstetric rapid response team. Finally, Drs Briggs and Peitzman discuss surgical rapid response teams. While the authors hope that readers will be able to emulate them at their organization, we also hope that readers will find commonalities in the RRS approach and apply those principles to other situations.

Dr Hillman and I share another desire: nobody should die in hospital unexpectedly. The cardiac-arrest-rate goal should be zero. We recognize that of course some patients in hospital will and must die. For those patients, the deaths should be expected, prepared for, and treated with care and comfort.

Hospital acuity is increasing, and the process of care is increasingly complex. The need for reorganization of hospital care into clusters of goal-directed rapid response teams is becoming more apparent. We may be near the tipping point for this reorganization.

Michael A. DeVita, MD, FCCM, FRCP, FACP Harlem Hospital Center 506 Lenox Avenue New York, NY 10037, USA

Kenneth Hillman, AO, MBBS, MD, FRCA, FCICM, FRCP Simpson Center for Safety

PO Box 3154

Liverpool, NSW 2170, Australia

E-mail addresses:

Michael.devita@nychhc.org (M.A. DeVita)

k.hillman@unsw.edu.au (K. Hillman) 